\title{
FILOSOFÍA Y CULTURA EN AMÉRICA LATINA
}



Fecha de entrega: 15 de octubre de 2011

Fecha de aprobación: 15 de noviembre de 2011

\title{
REFLEXIONES SOBRE CREATIVIDAD: EL PODER DE SUBJETIVACIÓN DEL SER HUMANO
}

\author{
REFLECTIONS ON CREATIVITY: \\ THE POWER OF HUMAN SUBJECTIVITY
}

Jochen Dreher*

\section{Resumen}

La creatividad especialmente surge del poder de subjetivación y de trascenderse a sí mismo el ser humano. La capacidad de ser creativo resulta, desde la perspectiva de la antropología filosófica, de la "posicionalidad excéntrica" del ser humano, la cual explica su poder de reflexividad y su compulsión y necesidad de crear cultura. Según la teoría de la creatividad de orientación sociológico-antropológica de Heinrich Popitz, esta capacidad humana de desarrollar lo nuevo está basada en las formas de acción de explorar, crear, generar sentido y jugar. Para explicar el poder de subjetivación del individuo, introducimos la sociología con orientación fenomenológica de Alfred Schütz, quien ofrece una teoría de las relevancias que permite analizar las motivaciones subjetivas que conducen el proceso creativo.

\section{Palabras clave}

Creatividad, subjetivación, imaginación, conocimiento del mundo de la vida, relevancias.

* Desde 2005 es director del Archivo de Ciencias Sociales de la Universidad de Konstanz (Alemania). Es docente de la Universidad de Konstanz y de la Universidad de St. Gallen. El presente artículo hace parte de los proyectos y temas de investigación que el autor desarrolla en campos como la sociología del conocimiento, la fenomenología y la sociología de la cultura, las organizaciones y la migración. Correo electrónico: Jochen.Dreher@uni-konstanz.de 


\begin{abstract}
Creativity specifically emerges from the power of subjectivation and self-transcendence of human beings. From the perspective of philosophical anthropology, the capacity to be creative results from the "eccentric positionality" of humans beings, which explains their power of reflexivity and the compulsion and necessity to create culture. According to the sociological-anthropological theory of creativity by Heinrich Popitz, this human capacity to develop something new is based on the forms of action of exploring, creating, establishing meaning and playing. To explain the power of subjectivation of the individual, we introduce the phenomenologically oriented sociology of Alfred Schütz, who offers a theory of relevances which allows analyzing the subjective motivations which control the creative process.
\end{abstract}

\title{
Keywords
}

Creativity, subjectivation, imagination, life-worldly knowledge, relevances.

\section{Introducción}

Según la argumentación del sociólogo Heinrich Popitz, el condicionamiento cultural y la relatividad de las normas sociales pueden verse desde dos perspectivas diferentes. Por una parte, se puede describir la plasticidad social del ser humano: su maleabilidad, su capacidad de reacción a diferentes tipos de órdenes sociales; y por otra parte, su productividad social, expresada en el poder de creación e imaginación, con las cuales los seres humanos conciben su vida social, interpretan hechos biológicos, transforman condiciones y se forman a sí mismos a través de su comportamiento. Estos dos aspectos de los seres humanos se desafían entre ellos (Popitz, 2006: 63 y ss.). De las reflexiones de Popitz surge una teoría de la creatividad que propone una confrontación flexible del ser humano con las normas sociales, y que está basada tanto en la productividad individual y social del ser humano como en su potencial de trascenderse a sí mismo.

Con creatividad quiero describir, siguiendo a Popitz, la capacidad humana de desarrollar lo nuevo. La creatividad se refiere a las formas de acción de explorar, crear, generar sentido y jugar. El explorar se dirige a obtener o generar conocimiento nuevo; es una forma de acción teórica que tiene como motivación cognitiva el iluminar lo desconocido. El conocimiento nuevo surge de explorar, es decir, de buscar, probar, preguntar, descubrir o inventar. "Explorar" es una expresión del poder de subjetivación del ser humano porque resulta de su capacidad de imaginar realidades para sí mismo. Crear, en cambio, es la objetivación intencional de lo imaginado, que sirve para la producción creativa de artefactos técnicos y estéticos. Por eso, en la creación se manifiesta el poder de $o b$ jetivación del ser humano (Pohlmann, 2006: 43). La capacidad del ser humano de generar sentido, como otra forma de acción de la creatividad, se refiere al poder de trascender del ser humano, cuyo mundo se presenta como un mundo de sentido que hay que interpretar. El ser humano puede trascender su mundo de la vida cotidiana, por ejemplo, construyendo mundos religiosos imaginarios. Jugar, por el contrario, no produce nada que persista; jugar puede dejar sin efecto, momentáneamente, 
motivos y estructuras del mundo de la vida cotidiana, y encuentra su sentido en sí mismo (Pohlmann, 2006: 44; Popitz, 2000: 92 y ss.). Específicamente el poder de subjetivación del ser humano que se manifiesta en el explorar demuestra el potencial del individuo para distanciarse de normas y roles impuestos, porque el poder de subjetivación resulta de evaluaciones y decisiones individuales que definen si la acción respeta o rechaza las normas sociales.

Partiendo de las reflexiones de la sociología antropológica de Heinrich Popitz, deseamos establecer un puente teórico con la sociología de orientación fenomenológica de Alfred Schütz. La meta es describir el poder de subjetivación del ser humano que sirve para crear lo nuevo, para la creatividad. Después de una breve presentación de la teoría de la creatividad de Popitz, su argumentación será confrontada con la teoría de las relevancias de Schütz, para finalmente proponer un análisis de la capacidad antropológica de la creatividad.

\section{La teoría de la creatividad de Heinrich Popitz}

Si partimos de la perspectiva de la sociología antropológica, la tensión entre conformidad a las normas y libertad de acción resulta de la constitución biológica del ser humano, quien está obligado a construir su entorno con las acciones para satisfacer sus necesidades vitales (Popitz, 2010: 87). La constitución biológica no prescribe cómo organizar el entorno, porque el ser humano es independiente de su instinto y puede reaccionar a sí mismo sin acudir a él, sin saber instintivamente cómo tiene que actuar y sin conocer los límites dentro los cuales puede actuar. Estas reflexiones tienen como base, entre otras teorías, la antropología filosófica de Helmuth Plessner, quien parte de la concepción de una "posicionalidad excéntrica" del ser humano, refiriéndose a la autorreflexividad del sistema de vida humana (Plessner, 2003: 360 y ss.). Con la expresión "excentricidad" Plessner se refiere a la forma posicional propia y exclusiva del ser humano, la cual constituye la categoría fundamental de su pensamiento antropológico. Excentricidad es la capacidad de distanciarse de sí, de aprehender objetos y realidades consistentes y de vivenciarse a sí mismo como persona, en cuanto al mundo personal de los semejantes y en cuanto al mundo apersonal de puros objetos.

Las capacidades de poder concebirse a sí mismo como persona y a las apariencias fenoménicas (Erscheinungen) como cosas (Dinge) son poseídas exclusivamente por el ser humano (González Jara, 1971: 137). El concepto de "posicionalidad excéntrica" está basado en la idea de que el ser humano tiene una "realidad somática" (Leib) y, al mismo tiempo, es cuerpo (Körper). El cuerpo del ser humano como centro de la posicionalidad tiene, además, una distancia con la "realidad somática", por lo que se posibilita la autorreflexividad y la autoconciencia. Por su forma de vida excéntrica y por su necesidad de compleción, el ser humano está obligado a crear cultura "artificialmente" a través de sus acciones (Dreher y Walter, 2010: 295). Por eso, según Plessner, la cultura se puede describir como la "segunda naturaleza" del ser humano (Plessner, 2003: 284). Por su forma de existencia, por su naturaleza, el ser humano es artificial, no se encuentra en un equilibrio y continuamente debe adaptarse al lugar y al tiempo en que se encuentra (Dreher, 2008: 1148). La circunstancia específica de la "artificialidad natural" establece la base de lo que Heinrich Popitz describe como la "ineludible sociabilidad de la acción humana”, que resulta de la compulsión de crear cultura (Popitz, 2010: 17). Estas reflexiones que parten de la antropología filosófica sirven 
pues a Popitz para desarrollar una teoría de la creatividad ${ }^{1}$.

La capacidad - poder-de imaginación del ser humano no se encuentra ligada a lo experimentado, sino que también llega más allá de lo que "realmente" pasa. Nuestra segunda realidad, la realidad de la imaginación, es más amplia que la realidad de nuestra percepción. El poder de la imaginación consiste en la capacidad de realizar lo alternativo; es una capacidad de concebir contrarrealidades. A Popitz no le interesan los procesos psíquicos de la producción creativa, sino lo producido: las realidades producidas por la imaginación, el mundo humano como mundo creado de imaginaciones (Popitz, 2000: 83).

Cuando las ideas consisten en visualizaciones gráficas de los ausentes, se puede entender a la imaginación como una representación de lo invisible, como una penetración en lo oculto. La imaginación supera a las ideas convencionales en tanto trae a la memoria "algo" que no solo estaba ausente, sino también cuya aparición se había retirado. La señalización de la imaginación a través de la metáfora esto es, la penetración en lo oculto- parece más apropiada que cualquier fijación rígida; bienvenida es la libre asociación. La imaginación puede revelar algo velado, puede abrir algo cerrado. Ella nos puede introducir en nuevos campos de experiencias. Esto no se debe, bajo ningún concepto, a un acto de genialidad. También el niño que se pone a jugar el papel de la madre, adquiriendo de este modo una nueva perspectiva, hace un descubrimiento clave que le habilita un acceso a nuevas experiencias.

1 Los siguientes parágrafos contienen un resumen de la teoría de la creatividad de Heinrich Popitz, que sirve luego para una discusión de cara a las reflexiones epistemológicas de la fenomenología.
Popitz se pregunta cuándo un concepto ha penetrado suficientemente en lo oculto como para que podamos llamarlo "imaginación". Es casi imposible trazar límites precisos aquí. Para él no se trata de separar la paja del trigo; esto puede no resultar sencillo. Más bien se concentra en que una experiencia fundamental del ser humano es que lo esencial de sí mismo, de su existencia, es invisible e impalpable. La muerte, la procreación, el pensamiento, los sentimientos son procesos que tienen lugar bajo la piel. Y también ocurre lo mismo con lo esencial en otras personas y en la naturaleza: lo que percibimos sensorialmente a menudo adquiere su significado más profundo recién cuando nos imaginamos lo que está "más allá" o "allí dentro". Pero lo oculto adquiere su dignidad no solo por los límites de la percepción; también nuestra imaginación se encuentra con resistencias. Es como si el motor del mundo en el que vivimos se cerrara a nuestra imaginación, se le escapara. No logramos -no siempre logramos- tener éxito en dejar reposar a este escaparse del motor del mundo respecto de la imaginación. La oscuridad es un desafío a la conciencia humana, al que respondemos con una cualidad específica de esta: la imaginación (Popitz, 2000: 92 y ss.).

Popitz se concentra en los caminos de la imaginación, en los cuales están en juego bosquejos básicos de la existencia humana (Dasein). Los caminos de la imaginación determinan cómo las personas organizan su existencia. Esta condición la cumplen a través de tres vías de la imaginación: 1) La imaginación exploratoria es la que busca, prueba, pregunta, descubre e inventa; consiste en una penetración en lo oculto como penetración en realidades aún desconocidas: la búsqueda de conocimiento. 2) La imaginación creadora -la imaginación constructora del objeto- es mostrante, busca la producción y formación de artefactos; consiste en una penetración en lo oculto como una penetración en lo informe: 
la búsqueda de contenidos y maneras de efectuar. 3) La imaginación generadora de sentido es imaginación interpretante, fundamentante, justificante; consiste en una penetración en lo oculto como una penetración en lo sensorial no perceptible: la búsqueda de sentido.

El mundo se le aparece al ser humano para ser explorado, creado, para darle sentido, ser interpretado. Estos son modos igualmente originarios de la relación del ser humano con el mundo y no es posible derivar unos de otros. Así, los supuestos de prioridad filogenética no son fundamentables.

La imaginación exploratoria comienza con la curiosidad del niño, con la búsqueda y recolección de él, con las primeras exploraciones con las manos en las primeras etapas de la humanidad. Al explorar el mundo tratamos de dar un nuevo paso en la familiaridad con él. En tanto descubrimos lo que a la vida le es útil y lo que le es hostil, cambiamos la base del cuidado de la existencia humana. Pero la búsqueda de conocimiento no se limita al conocimiento de la naturaleza: la imaginación exploratoria puede sondear también hechos sociales y hacer comprensible de una nueva forma la estructura de la convivencia humana. Y ella puede, no en último término (¿o tal vez sí en último término?), conducir a una nueva comprensión de las emociones humanas al penetrar en la oscuridad del yo explorador.

La imaginación creadora se basa en la capacidad el actuar objetivante del hombre con miras a la objetivación de sus intenciones. Esto rige tanto para la técnica como imaginación creadora de la producción de artefactos, como para el arte en tanto imaginación creadora de la formación de artefactos (incluyendo la formación de sonidos y palabras). El hecho de que yo aquí las una y resuma se justifica por la base que tienen en común: la capacidad humana para poner algo en práctica. Ambos casos se basan en la capacidad de intervención humana en lo informe, en la inteligencia biológicamente fundada del "hacer otra cosa" o "hacer algo diferente".

Por supuesto, la unión de técnica y arte no significa una renuncia a la distinción, por muy difícil que a menudo sea diferenciar los artefactos fabricados puramente por utilidad de un ambicionado valor de la forma. La estética ha llamado a tal valor de la forma "belleza" y "armonía", aunque sobre esto puede discutirse. Indiscutible es el hecho de que la aspiración a formas creadas corresponde a la condición humana. Al igual que la imaginación exploratoria, la imaginación creadora tampoco se limita a los objetos tangibles; también las instituciones como "artefactos sociales" pueden ser creadas de forma innovadora. Una nueva forma de institucionalidad se encontraba en las primeras culturas agrarias con la división social del trabajo que surgió con la llegada de la artesanía, en la burocracia con la administración centralizada de las sucursales regionales y en la democracia ateniense con la ciudad-estado (Popitz, 2000: 95).

Explorar y crear están unidos por una variedad de interacciones. Sin embargo, son de carácter opuesto en tanto constituyen respuestas humanas a las condiciones de su existencia. En el crear (en todas las variantes mencionadas) se pone de manifiesto la capacidad humana de exteriorizar ideas o representaciones y de construirlas en el mundo. En aquello que se vuelve forma se demuestra el poder de objetivación del ser humano. En el explorar se manifiesta la capacidad de transformación de la realidad conocida; explorando transformamos nuestra imagen de la realidad. Que seamos capaces de ello, como argumenta Popitz, demuestra la capacidad del sujeto cognoscente de construir realidades en su interior: el poder de subjetivación del ser humano. 
La imaginación generadora de sentido, en tanto va más allá de la búsqueda individual de este, se cristaliza en sistemas de creencias religiosas: los grandes sistemas imaginarios del mundo. A pesar de las diferencias, todos tienen en común que ubican el destino del ser humano en relación con algo no humano, en relación con fuerzas divinas. La experiencia religiosa es la experiencia de algo "fuera de". Un afuera que es superior a los seres humanos y que, a pesar de ello, no escapa del todo a su conocimiento, el cual es accesible a través de la magia, el sacrificio, la oración, el ascetismo, la meditación. En el proceso de secularización, los sistemas religiosos de creencias son puestos en cuestión por los sistemas de la filosofía y las ciencias, que engendran un nuevo tipo de generación de sentido (por propia voluntad o involuntariamente, "cubriendo necesidades"). Los puntos de inflexión más graves en la historia europea de la racionalización fueron la filosofía griega de la naturaleza y el nacimiento de las ciencias naturales modernas en el siglo XVII. Así como el poder de objetivación se manifiesta en la imaginación creadora y el poder de subjetivación se materializa en la imaginación exploratoria, así también se realiza el poder de trascendencia del hombre en la imaginación generadora de sentido (Popitz, 2000: 96 y ss.).

Los poderes de la exploración, la creación y la generación de sentido son abiertos entre sí; pueden unirse en una gran variedad de formas sin perder su individualidad, reforzarse y obstaculizarse, superponerse o atravesarse. El niño que apila los bloques de construcción, uno encima del otro, logra con la imaginación creadora un nuevo artefacto, y explora, al mismo tiempo, cuán alta puede ser la torre sin que se caiga. En las conexiones de las diferentes vías de la imaginación, la imaginación lúdica tiene un significado especial. En la efímera creatividad del juego inventamos "realidades no realistas", algo real que, simultáneamente, es ficticio: inventamos una realidad "como si fuera".

No he contado a la imaginación lúdica dentro de las tres formas básicas debido a que ella no produce nada duradero, ningún conocimiento nuevo, ninguna forma permanente, ningún sentido interpretativo. El juego se agota en el hacer lúdico, aunque la imaginación lúdica puede inspirar algo permanente cuando se traduce en innovaciones de la exploración, la creación y la generación de sentido. Esto sucede a menudo de forma maravillosa, como en la actuación de las fiestas de culto, en la variación lúdica de formas arquitectónicas, en la ironía de las narraciones de fábula. Así como la exploración, la creación y la generación de sentido están abiertos unos a otros, también están abiertos a la vagabunda imaginación del juego (Popitz, 2000: 97).

Lo que producimos de manera creativa está movido por la imaginación. Sin duda, otras cosas forman parte del éxito creativo: la perseverancia, la habilidad, la suerte; pero la imaginación es una condición necesaria. La creatividad es un acto que trae a la existencia (Dasein) algo que antes no existía. Eso no quiere decir que ese "algo" tenga que caer del cielo sin precedentes, pero podemos producir algo que es más que mera repetición, confirmación y acrecentamiento de lo dado. La creatividad es la capacidad de ser autor. Debido a que las personas pueden ser creativas, son capaces de alterar activamente las condiciones de su existencia a partir de sus propias fuerzas. En el caso de la exploración y la generación de sentido, la creación producida es cognitiva (un nuevo conocimiento, nuevas certezas de creencias); en el caso de la creatividad, ella es fáctica, artefáctica.

Según la argumentación de Popitz, no todo lo que exploramos o creamos, no cada sentido que encontramos es creativo. A la imaginación, para penetrar en lo oculto, 
le hace falta algo más: debe tener éxito en conseguir desde lo oculto algo innovador, en encontrar algo nuevo en lo oculto. Explorar, crear, generar sentido son caminos hacia la autoría. Pueden ser creativos cada uno de una manera especial, sin embargo, ¿existen también cosas en común entre la creatividad de los diferentes caminos de la imaginación? Popitz creía que al menos una cosa en común se puede encontrar. El ser humano es creativo fundamentalmente debido al hecho de que es capaz de captar "lo existente fuera de su yo" como "una alteridad, un ser así peculiar". Comprender el sentido particular del ser otro es parte del ser particular del ser humano.

Podemos enfrentarnos al mundo con interés alocéntrico; lo podemos concebir en términos de lo que es diferente y está fuera de nosotros mismos. La base de este logro es la independencia de la imaginación respecto de la percepción. Que podamos desprendernos del mundo dado de la percepción y podamos penetrar de forma imaginativa en lo oculto nos pone en una relación especial respecto a la alteridad del ser fuera de nuestro ser: exploramos lo que nos está vedado, creamos lo que está más allá de la propia existencia volviéndola una realidad propia y producimos así un interlocutor objetivo e independiente; nos ponemos en contacto con lo no-yo (NichtSelbst), con lo oculto, relacionándonos con fuerzas divinas. El poder de imaginación de la fantasía nos transporta más allá de nosotros mismos a otra realidad que podemos identificar y reconocer como "la otra".

Esto contradice una fórmula común: "Hay una tendencia universal de la humanidad" -cita Popitz a Hume-, y Freud se refiere a eso, con aprobación, como "a ver a todos los demás seres a uno mismo y transferir las propiedades a ellos, con las cuales uno mismo se siente familiarizado". Para Hume esto explica la propensión a la reanimación fantasmal de lo inanimado. En el animismo, especialmente, la propia experiencia de la dualidad del cuerpo y la mente se transmite a todos los objetos del mundo externo. Spencer, Bergson y muchos otros han adoptado la tesis de la transmisión. Freud advierte contra la idea de desvalorizar al animismo "la primera cosmovisión de la humanidad" como primitivismo superado. Todavía hoy las ideas animistas son virulentas no solo en tanto que superstición, sino "como el fundamento de nuestra habla, de nuestra creencia y de nuestro filosofar" (Popitz, 2000: 99 y ss.).

De hecho, la tendencia a transferir de manera antropomórfica las experiencias del yo (Selbsterfahrungen) a experiencias del mundo (Welterfahrungen) está en todas partes. En tanto que inclinación del sentir y pensar del ser humano, esta tendencia puede denominarse "universal". Sin embargo, los seres humanos pueden liberarse de este velo antropomórfico de sus experiencias en el mundo. No importa cuán fuertes sean las ataduras categóricas de nuestra capacidad de entendimiento, para Popitz existe la posibilidad de un giro hacia el exterior del espíritu humano, de abrirse a un no-yo, a otra voluntad, a otras necesidades, a otras condiciones de existencia. La singularidad potencialmente dialógica de la relación humana con el objeto (menschliches objektverhältnis) abarca un poder escuchar y ver no guiados antropocéntricamente, un entender "desde la perspectiva del otro". Nosotros somos capaces de dejarnos hacer preguntas por parte de las cosas de este mundo. Para la antropología, a la lógica del anthropos pertenece la habilidad de una visión no antropomórfica del mundo: la habilidad del alocentrismo.

Es sobre todo esta habilidad la que permite al ser humano ir más allá de lo ya existente. Porque es capaz de indagar en la otredad, la alteridad, the otherness de lo que existe fuera de él, porque puede llevar a cabo este viraje descentralizante, el ser humano está en la 
situación de no sumergirse en la circunstancia dada y mirar el mundo desde la luz de lo otro posible. La categoría de la alteridad lo torna creativo.

Como ejemplo servirá el alocentrismo del explorar. A continuación, primero una observación sobre la peculiar y alocéntrica imaginación del jugar. La estructura dialógica es común a todas las formas de jugar (juegos de roles, juegos de fantasía, juegos con reglas). Jugar es siempre un jugar con algo, con otro sujeto, con otra cosa, también con un compañero imaginario. El mero saltar, correr, mecerse también genera diversión, pero no constituye un juego, en tanto que no se hace referencia a un compañero y, de este modo, no se mantiene la condición dialógica.

No solo el jugador es activo, sino también aquello con lo que juega. El niño aprende desde temprano a atribuir actividades a las cosas con las que juega, para establecer una estructura dialógica: las hace hablar, obedecer o desafiar, reír y llorar, dormir y despertar. Jugar no significa solamente "jugar con algo", sino también que "algo juega con los jugadores". La estructura dialógica del juego no es, entonces, algo forzado, que se le impone; es inherentemente el modelo de acción del juego. Cuando jugamos estamos con un otro, estamos confrontando con un otro que responde. Esta confrontación adquiere su estímulo a través de la imprevisibilidad de la pareja de juego. Cómo actúa el contrincante, cómo responde-qué pieza mueve, a dónde, hacia dónde va la pelota, etc.- no se puede predecir con certeza. En la dinámica del juego se halla "básicamente un elemento de sorpresa", de "aventura" y de "ocurrencia". En particular, los juegos de competición o partidos son a menudo encarados para engañar o desorientar intencionalmente al contrincante. Sin la artimaña, la gracia de muchos juegos se pierde (Popitz, 2000: 101).
El momento de la sorpresa reclama una mayor concentración en el compañero de juego, en sus peculiaridades, en sus voluntariedades. La anticipación apropiada de aquello que es difícil de predecir pertenece a la razón del juego. En el juego sobreviene, comparado con situaciones similares -como la competencia económica-, la liberación de las presiones de la vida cotidiana. Jugar es una acción sobrante. Jugando hacemos algo lujoso, nos liberamos de los impulsos como el hambre y el miedo; también nos liberamos de la indomable sexualidad. El juego amoroso, que Buytendijk describe como "el ejemplo más puro de todos los juegos", se rompe, según Popitz, cuando la "domesticación" falla. Quien juega, juega voluntariamente; la coacción ahoga la predisposición a jugar.

El diálogo, la sorpresa, la acción sobrante aquí ponen en escena una concentración, particularmente alivianada y única, en los otros juegos de tipo intensivo. Esto ofrece la posibilidad al jugador de una inusual entrega a un no-yo. No podemos jugar intensamente si no podemos comunicarnos intensamente con la singularidad de aquel con quien jugamos. Especialmente en los juegos con objetos como contrapartes se puede llegar al ensimismamiento abnegado en la singularidad del oponente.

En un ejemplo de Popitz: un niño mueve piedras de colores de aquí para allá; deja balancearse a una pieza sobre un motivo de la alfombra; desparrama arena en su ombligo, una y otra vez; las olas que llegan a la playa pueden romperse con los pies, saltarse, ser evitadas por medio de una rápida retirada o desviadas de su propio sentido, gracias a la versatilidad de su forma; la arcilla que se comprime con la mano se modifica en un sin fin de transformaciones, de una figura a otra. La atracción especial de la experiencia alocéntrica del juego radica en la liviandad no obligatoria, en la fugacidad de su profundidad 
de pensamiento. Le debemos esta experiencia a nuestra capacidad de inventar un mundo más allá de lo que es "la realidad en serio" (Popitz, 2000: 103).

Después de analizar los fundamentos antropológicos de la creatividad siguiendo a Popitz, queremos introducir otra posición teórica, también en la tradición del individualismo metodológico, con la cual el factor de la subjetividad del actor individual se puede investigar en relación con la creatividad: la sociología con orientación fenomenológica de Alfred Schütz.

\section{Fenomenología de la relevancia: subjetivación y creatividad}

Si para un análisis de la creatividad nos concentramos en una sociología del conocimiento con base fenomenológica, tenemos que enfocar la relación dialéctica entre individuo y sociedad (Berger y Luckmann, 1993). Todo conocimiento derivado socialmente es aceptado por los miembros individuales de un grupo cultural como incuestionablemente dado. Esto es así porque este conocimiento es transmitido como válido y es ampliamente aceptado por los miembros del grupo; por consiguiente, se transforma en un esquema común de interpretación del mundo y en un medio de comprensión y de acuerdo mutuo.

Estas reflexiones que surgen de la sociología de Schütz están estrechamente conectadas con su concepción de la estructuración del conocimiento del mundo de la vida. Este último debe entenderse como el mundo preteórico de la experiencia; el mundo de la vida describe cierto estrato de la experiencia humana que es previo al mundo sociohistórico, con sus estructuras invariantes, las cuales son propias de todos los actos de conciencia humana (Luckmann, 1983: 41). Schütz formuló una versión modificada del concepto de mundo de la vida a partir de la fenomenología de Edmund Husserl, quien descubre la estructura general perteneciente al mundo de la vida en todas sus relatividades, donde todo Ser relativo está ligado a esta estructura general, la cual en sí misma no es relativa (Husserl, 2009).

Este concepto de mundo de la vida fue sociológicamente ampliado por Alfred Schütz, quien incluyó en él al mundo social y a las esferas de realidad extra-cotidianas. Es exactamente esta tipicidad la que Schütz toma en consideración al argumentar que el mundo intersubjetivo del actuar, en su totalidad en el tiempo estándar, y el problema de cómo se experimenta a otros seres humanos en la actitud natural no solo es un tópico de reflexión teórica, sino que es la cuestión central de las ciencias sociales (Schütz, 1995: 234 y ss.). Para nuestro análisis de la creatividad necesitaremos investigar específicamente el conocimiento del mundo de la vida del actor individual.

Si tenemos en cuenta el conocimiento del mundo de la vida y la estructura de la experiencia individual, es posible afirmar que los correlatos subjetivos de este conocimiento están vinculados a distintas zonas de motivación individual. El individuo que vive en el mundo siempre se experimenta a sí mismo en una situación que él o ella han definido. Esta situación está marcada por dos momentos diferentes: uno se origina en la estructura ontológica del mundo pre-dado; el otro componente resulta del estado biográfico real del individuo. El componente ontológico de la situación del individuo es experimentado como impuesto, sin la posibilidad de cambio espontáneo. Sin embargo, la situación biográfica determina las definiciones espontáneas de la situación dentro del marco ontológico impuesto (Schütz, 1970: 122).

Partiendo de estas reflexiones acerca de la objetividad dada de la estructura del mundo 
de la vida y de la situación biográfica subjetivamente experimentada, Schütz describe dos sistemas de relevancia de acuerdo a los cuales nuestro conocimiento está estructurado: diferencia entre sistemas de relevancia intrínsecos y sistemas de relevancia impuestos (Schütz, 2003: 126 y ss.). Las relevancias intrínsecas son el resultado de nuestros intereses elegidos, que son establecidos por nuestra decisión espontánea de resolver un problema mediante el pensamiento, de alcanzar un objetivo mediante nuestra acción o de concretar un estado de cosas ya proyectado. En oposición, las relevancias impuestas no se vinculan con intereses elegidos por nosotros, no derivan de actos de nuestro albedrío; debemos tomarlas tal como se nos presentan, sin el poder de modificarlas por nuestras actividades espontáneas.

Dentro de la tensión entre sistemas de relevancia intrínsecos e impuestos surge la creatividad del individuo. Esta es la expresión de la subjetividad del actor individual que tiene el poder de trascenderse a sí mismo. Mencionamos ahora tres tipos ideales de relevancias que desarrolla Schütz: "motivacionales", "temáticas" e "interpretativas", dejando claro que la expresión pura de estos tipos no puede encontrarse empíricamente. Con el término "relevancias motivacionales" Schütz describe el interés experimentado subjetivamente como el motivo para la definición de una situación, independientemente de qué componentes de la situación biográfica sean impuestos o si estos son el resultado de nuestra propia espontaneidad (Schütz, 1970: 123 y ss.). Todas las relevancias motivacionales son también subjetivamente experimentadas como un sistema de planes en el marco de nuestro plan de vida. En este sentido, las relevancias motivacionales sirven como base de la imaginación exploratoria, porque en la base de un conocimiento subjetivo del individuo dirigen su búsqueda, su descubrimiento y su invención. Si queremos explicar cómo surge el conocimiento nuevo en el proceso creativo, tenemos que dedicarnos al próximo tipo de relevancias.

Las "relevancias temáticas", sin embargo, surgen de la circunstancia en la que no todos los elementos motivacionalmente dados son suficientemente familiares o previamente conocidos. También puede suceder que la situación actual no coincida con el tipo de situación presente en el propio acervo de conocimiento, de ahí que se requiera un conocimiento suplementario vinculado a la situación; esto significa un conocimiento adicional y relevante para la definición de la situación. De esta surge el aspecto de la acción creativa que describimos como "explorar", que intenta generar conocimiento nuevo iluminando lo desconocido. Persiguiendo intereses subjetivos, el individuo, con su capacidad de imaginación, puede desarrollar realidades que se diferencian de lo dado y que ofrecen la posibilidad de continuar con la acción. Es exactamente este potencial del actor individual de establecer evaluaciones y decisiones el que expresa su poder de subjetivación. En la base de la conciencia subjetiva, el individuo puede construir con su imaginación mundos divergentes de la realidad actual, que ofrecen soluciones dentro de la acción confrontada con obstáculos.

En relación con el tercer tipo ideal, las "relevancias interpretativas", podemos discutir lo que estuvimos describiendo como "crear" y "generar sentido", en relación con el poder de objetivación y de trascenderse a sí mismo del ser humano individual.

Estas relevancias aportan a la solución de las cuestiones temáticamente destacadas. Algunos elementos del conocimiento son útiles para la interpretación, ya que se establecen procesos de tipificación que resultan en la objetivación de conocimiento nuevo. El individuo, persiguiendo sus intereses y 
motivaciones subjetivamente establecidas, realiza el proceso de la acción creativa desarrollando tipificaciones, pero también artefactos técnicos y estéticos nuevos. Del poder de subjetivación en el proceso creativo resulta el poder de objetivación que puede tener como resultado la obra de arte, adelantos técnicos o intelectuales, etc.; resumiendo, tiene como resultado la creación cultural basada en la reflexividad específica del ser humano.

\section{Referencias}

Berger, P. y Luckmann, T. (1993). La construcción social de la realidad. Buenos Aires: Amorrortu.

Dreher, J. (2008). Über die "natürlichen" Grenzen der Sozialwelt. Phänomenologische und philosophisch-anthropologische Grundlegungen der Soziologie. En Die Natur der Gesellschaft. Verhandlungen des 33. Kongresses der Deutschen Gesellschaft für Soziologie in Kassel 2006, Teilband 2. Nueva York: Campus.

Dreher, J. y Walter, M. (2010). Nachwort. En Einführung in die Soziologie. Konstanz: Konstanz University Press.

González Jara, A. (1971). La posicionalidad excéntrica del hombre. Anuario Filosófico, 4(1), pp. 117-181.

Husserl, E. (2009). La crisis de las ciencias europeas y la fenomenología trascendental. Buenos Aires: Biblos.
Luckmann, T. (1983). On the Boundaries of the Social World. En Life-World and Social Realities. Londres: Heinemann.

Plessner, H. (2003). Die Stufen des Organischen und der Mensch. Einleitung in die philosophische Anthropologie. Frankfurt: Suhrkamp.

Pohlmann, F. (2006). Heinrich Popitz - sein Denken und Werk en Soziale Normen. Frankfurt: Suhrkamp.

Popitz, H. (2000). Wege der Kreativität. Tübingen: Mohr Siebeck.

Popitz, H. (2006). Soziale Normen. Frankfurt: Suhrkamp.

Popitz, H. (2010). Einführung in die Soziologie. Konstanz: Konstanz University Press.

Schütz, A. (1970). Some Structures of the Life-World, in Collected Papers. Vol. III: Studies in Phenomenological Philosophy. La Haya: Nijhoff.

Schütz, A. (1995). Sobre las realidades multiples. En El problema de la realidad social. Escritos I. Buenos Aires: Amorrortu.

Schütz, A. (2003). El ciudadano bien informado. Ensayo sobre la distribución social del conocimiento. En Estudios sobre teoría social. Escritos II. Buenos Aires, Madrid: Amorrortu. 